\title{
Etude ethnobotanique des plantes à activités antiparasitaires utilisées en médecine traditionnelle dans la préfecture de Doufelgou au nord du Togo.
}

\author{
Iwaba Kpabi*, Amégninou Agban1, Yao Hoekou1, Passimna Pissang1, Tchadjobo Tchacondo1, \\ Komlan Batawila ${ }^{2}$ \\ ${ }^{1}$ Laboratoire des Sciences Biomédicales, Alimentaires et de Santé Environnementale (LaSBASE), Ecole Supérieure \\ des Techniques Biologiques et Alimentaires (ESTBA), Université de Lomé, Togo. \\ 2Laboratoire de Botanique et Ecologie Végétale, Faculté des Sciences (FDS), Université de Lomé, Togo. \\ Correspondance : kpabi2010@gmail.com
}

Original submitted in on 20 th December 2019. Published online at www.m.elewa.org/journals/ on $30^{\text {th }}$ April 2020 https://doi.org/10.35759/JABs.148.2

\begin{abstract}
RESUME
Objectif : L'objectif de ce travail a été de recenser et d'identifier les plantes utilisées par les tradipraticiens dans le traitement des maladies parasitaires au Togo.

Méthodes et Résultat : L'enquête a été effectuée à l'aide d'un questionnaire semi-structuré auprès de 34 tradipraticiens de juin à septembre 2017 dans la préfecture de Doufelgou au nord du Togo. Les données ont été traitées à l'aide du tableur Excel 2013. A l'issue de cette enquête, 50 espèces appartenant à 28 familles et 46 genres ont été recensées. Les plantes les plus citées pour leur utilisation contre le paludisme étaient : Newbouldia laevis (26,47\%), Aloe buettneri $(17,64 \%)$ et Sarcocephalus latifolius (11,76\%). Euphorbia hirta $(38,23 \%)$, Cassia sieberiana $(32,35 \%)$ et Carica papaya $(26,47 \%)$ ont été citées pour leur utilisation contre les nématodes. Pour le traitement de la dysenterie amibienne, Flueggea virosa $(26,47 \%)$ et Khaya senegalensis (14,70\%) étaient les plus citées. Les feuilles $(22,91 \%)$ et les écorces de tiges $(21,35 \%)$ étaient les parties les plus utilisées sous forme de décoction $(47,91 \%)$ et de macération $(40,62 \%)$. Les recettes sont administrées majoritairement par voie orale $(84,81 \%)$. Le facteur de consensus (FC) d'utilisation de ces plantes était élevé pour le traitement des maladies parasitaires étudiées (vers intestinaux, $F C=0,83$; paludisme, $\mathrm{FC}=0,63$ et amibiase, $\mathrm{FC}=0,56$ ).

Conclusion et application des résultats Cette étude révèle qu'il existe de réelles connaissances sur les plantes médicinales utilisées pour le traitement des maladies parasitaires au Togo et constitue une base de données pour des études ultérieures. Des études phytochimiques seront effectuées sur une sélection de ces plantes à la recherche de nouvelles molécules à activité anthelminthique.
\end{abstract}

Mots clés : Maladies parasitaires, plantes médicinales, tradithérapie, Togo. 
Kpabi et al., J. Appl. Biosci. 2020 Etude ethnobotanique des plantes à activités antiparasitaires utilisées en médecine traditionnelle dans la préfecture de Doufelgou au nord du Togo.

\title{
Ethnobotanical study of plants with antiparasitic activities used in traditional medicine in Doufelgou in northern Togo.
}

\begin{abstract}
Objective: The objective of this work was to identify the plants used by traditional healers in the treatment of parasitic diseases in Togo.

Methodology and Results: The survey was carried out using a semi-structured questionnaire with 34 traditional healers from June to September 2017 in the prefecture of Doufelgou in northern Togo. The data was processed using the Excel 2013 spreadsheet. At the end of this survey, 50 species belonging to 28 families and 46 genera were identified. The most cited plants for their use against malaria were Newbouldia laevis (26.47 \%), Aloe buettneri (17.64 \%) and Sarcocephalus latifolius (11.76 \%). Euphorbia hirta (38.23 \%), Cassia sieberiana (32.35\%) and Carica papaya (26.47 \%) were cited for their use against nematodes. For the treatment of amoebic dysentery, Flueggea virosa (26.47\%) and Khaya senegalensis (14.70 \%) were the most cited. Leaves $(22.91 \%)$ and stem barks $(21.35 \%)$ were the most used parts. These plant organs are mainly used as decoction (47.91\%) and maceration (40.62\%), and the recipes are administered orally (84.81 $\%)$. The consensus factor $(\mathrm{Cl})$ for the use of these plants was high for the treatment of the parasitic diseases studied (intestinal worms, $\mathrm{Cl}=0.83$; malaria, $\mathrm{Cl}=0.63$ and amoebiasis, $\mathrm{Cl}=0.56$ )

Conclusion and application of the results: This study reveals that there is real knowledge about medicinal plants used for the treatment of parasitic diseases in Togo and constitutes a database for further studies. Phytochemical studies will be carried out on a selection of these plants in search of new molecules with anthelmintic activity.
\end{abstract}

Key words: Parasitic diseases, medicinal plants, traditional therapy, Togo.

\section{INTRODUCTION}

Les maladies parasitaires constituent un problème de santé publique dans les pays en développement et particulièrement en Afrique. Le paludisme est l'une des principales maladies parasitaires tropicales responsables d'une morbidité et d'une mortalité importantes, en particulier chez les enfants et les femmes enceintes (Idowu et al., 2010). Chaque année, plus de 300 millions de cas de paludisme aigus sont relevés dans le monde, et près d'un à deux millions de personnes en meurent. Environ $90 \%$ de ces décès surviennent en Afrique (Lakouéténé et al., 2009 ; Idowu et al., 2010). Les helminthiases intestinales occupent également une place de choix au sein des maladies parasitaires dans le monde en particulier dans les pays en développement des régions tropicales (Menan et al., 1997). Dans ces zones, il est fréquent de rencontrer des sujets portant plusieurs parasitoses (Niaré et al., 2018). Les conditions climatiques, d'hygiène précaires et le niveau socio-économique faible font que ces parasitoses sont endémiques dans ces pays (Menan et al., 1997). La lutte contre le paludisme s'est longtemps basée sur l'utilisation des antipaludiques de synthèse (nivaquine, flavoquine, méfloquine etc.) et naturel (quinine) qui sont devenus moins actifs sur les métazoaires du Plasmodium (N'Guessan et al., 2009). Par la suite, l'utilisation des combinaisons thérapeutiques à base d'artemisinine a pris la relève. La lutte contre les géohelminthoses est faite par des traitements avec des anthelminthiques tels que le Praziquantel, l'Albendazole et l'Iververmectine (Niaré et al., 2018). Cependant, les parasites sont de plus en plus résistants à ces médicaments; il est donc nécessaire de rechercher continuellement de nouvelles molécules thérapeutiques. Les produits naturels représentent une source importante de nouvelles molécules actives car plus de la moitié des produits cliniquement prouvés sont d'origine naturelle (Danton et al., 2019). Les plantes médicinales sont une source de médicament et de nourriture pour la majorité de la population vivant dans les zones rurales des pays en développement comme le Togo où les ressources sont limitées 
(Karou et al., 2011 ; Kpodar et al., 2015 ; Adebayo and Amoo, 2019). Cette connaissance des propriétés des plantes qui a déjà fait ses preuves est basée sur une observation empirique et est transmise de génération en génération (Danton et al., 2019). Malgré l'utilisation presque exclusive des remèdes à base de plantes dans la lutte contre les maladies parasitaires dans plusieurs régions $d u$ Togo, peu de connaissances sont disponibles sur

\section{MATERIEL ET METHODE}

Zone d'étude: L'enquête a été réalisée dans la préfecture de Doufelgou. Elle est localisée dans la région de la Kara, située au Nord du Togo (entre la longitude $0^{\circ} 52^{\prime}$ et $1^{\circ} 23^{\prime}$ Est et la latitude $9^{\circ} 37^{\prime}$ et $10^{\circ} 03^{\prime}$ Nord). Elle jouit d'un climat tropical de type soudanien à régime unimodal qui se caractérise par une seule grande saison pluvieuse et une grande saison sèche. Les pluies s'étendent de juin à novembre avec un maximum en août. Les précipitations annuelles varient entre 1000 et $1800 \mathrm{~mm}$ et les températures moyennes mensuelles varient entre $24^{\circ} \mathrm{C}$ et $29^{\circ} \mathrm{C}$, avec une moyenne annuelle ces plantes à activités antiparasitaires et leurs utilisations par les populations indigènes. Les enquêtes ethnobotaniques sont donc essentielles pour fournir de nouvelles pistes pour les dépistages pharmacologiques. L'objectif de ce travail a été de recenser et d'identifier les plantes utilisées par les tradipraticiens dans le traitement des maladies parasitaires au Togo.

de $25 \mathrm{C}$. La préfecture de Doufelgou est peuplée de Nawd'ba et de Lamba, deux peuples différents sur le plan linguistique et artisanal que le colonisateur a regroupé sous le nom local "Losso". Estimée à 78635 habitants en 2010, la population de Doufelgou est constituée de ruraux à plus de $90 \%$. L'agriculture (dont les principales cultures sontle sorgho, le fonio, l'arachide, le niébé, le maïs, le manioc, l'igname, etc.) et l'élevage y sont les principales activités socioéconomiques (Koumantiga et al., 2013 ; Sema et al., 2018).

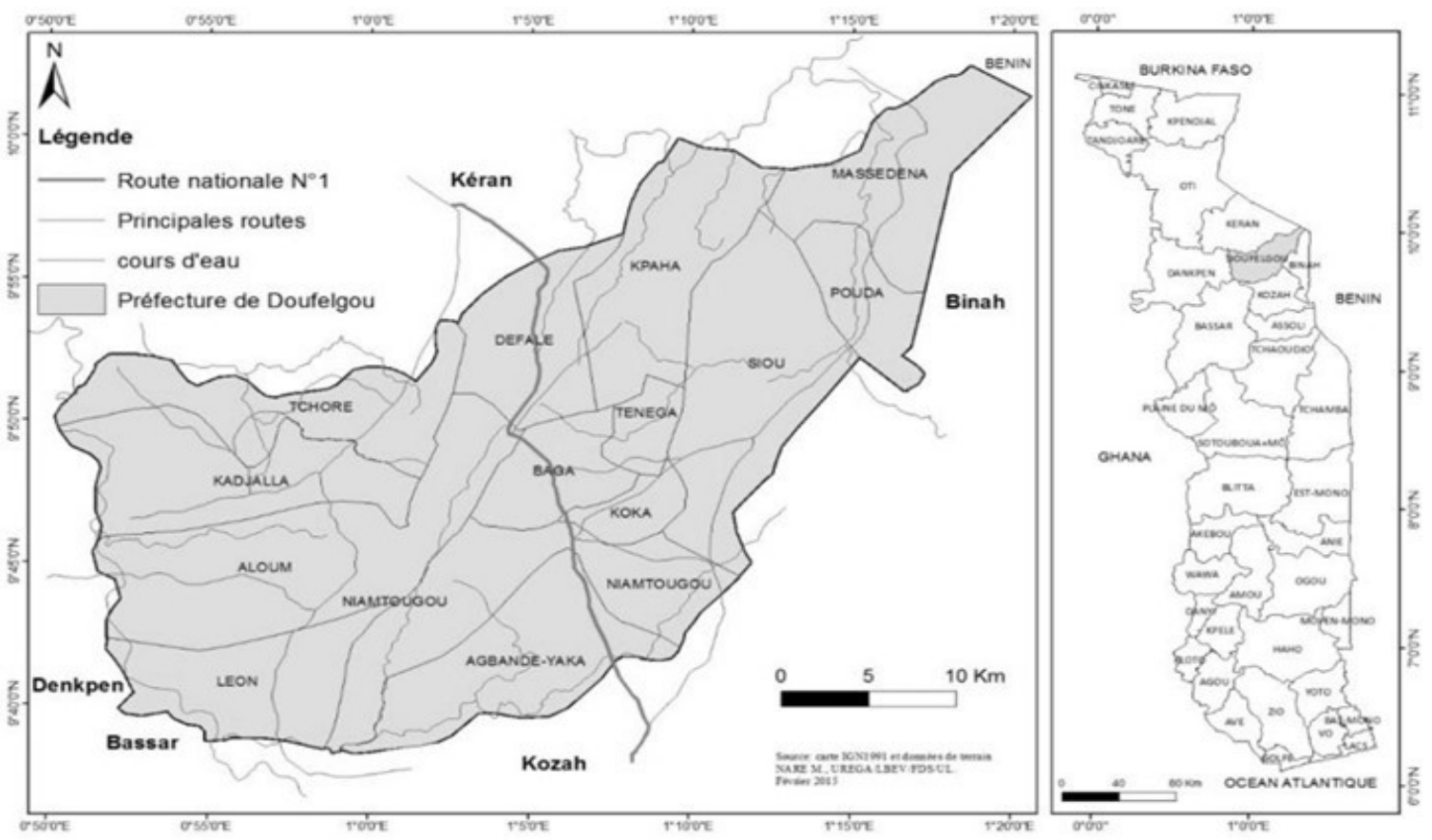

Figure 1 : Situation géographique de Doufelgou.

Enquête ethnobotanique: II s'agit d'une étude transversale descriptive réalisée dans la préfecture de Doufelgou entre juin et septembre 2017. La population de l'étude est constituée de tradithérapeutes et de personnes ressources habitant la préfecture au cours $d u$ déroulement de l'enquête. L'enquête ethnobotanique a été réalisée à l'aide d'un questionnaire semi-structuré. Le questionnaire a été axé sur les habitudes thérapeutiques de la population en matière de lutte contre les maladies parasitaires (Paludisme, Vers 
intestinaux, Amibiase), le nom local, les parties de la plante utilisée, les indications thérapeutiques, les méthodes de récolte, les recettes, les modes d'administration.

Identification des plantes: Après interrogation, une identification préliminaire est faite sur le champ. Après quoi, des photographies des plantes sont effectuées et un échantillon est collecté et conditionné pour identification au Laboratoire de Botanique et Ecologie Végétale de la Faculté des Sciences (FDS) de l'université de Lomé.

Traitement des données : Les données ont été saisies et traitées à l'aide de MS Excel (2013). La fréquence relative de citation (FRC) de chaque espèce a été calculée suivant la formule :

\section{RESULTATS}

Informations générales sur les personnes enquêtées : Les enquêtés sont à $70,58 \%$ de sexe masculin et $29,42 \%$ de sexe féminin. L'étendue d'âge des personnes interrogées allait de 26 à 80 ans avec une moyenne de 51,71 $\pm 13,23$ ans. La tranche d'âge 46-60 était la plus représentée avec $55,88 \%$. Parmi les personnes enquêtées $29,41 \%$ étaient analphabètes, $44,52 \%$ avaient un niveau secondaire et $2,94 \%$ un niveau universitaire (Tableau 1). Concernant l'origine du savoir, $51,61 \%$ des personnes enquêtés ont hérité la

$$
F R C=\frac{M}{N}
$$

avec $\mathrm{M}$ le nombre de personnes ayant cité la plante en question et $\mathrm{N}$ le nombre total de personnes enquêtées. Le résultat est ensuite exprimé en pourcentage (Ouachinou et al., 2019). Le facteur de consensus FC autour de ces pathologies pour les espèces recensées est déterminé par la formule suivante :

$$
F C=\frac{N-N e}{N-1}
$$

Où $\mathrm{N}$ est le nombre de fois que la maladie est citée et Ne le nombre total d'espèces citées pour la soigner. Les valeurs du FC varient entre 0 et 1 (Heinrich et al., 1998 ; Hoekou et al., 2016).

phytothérapie de leur ascendant tandis que 32,26 \% l'ont acquis par leur expérience. Les résultats montrent que $52 \%$ des personnes enquêtés font la phytothérapie de coutume, $36 \%$ le font à cause de son faible coût et $6 \%$ pensent qu'elle est plus efficace que la médecine moderne (Tableau 1). Plus de la moitié des personnes enquêtées (52\%) coopère avec les médecins. Le groupe ethnique des enquêtés était Lamba $(50,00 \%)$, Nawd'ba (47,06 \%) et Kotokoli (2,94\%).

Tableau 1. Données sociodémographique des personnes enquêtées

\begin{tabular}{|l|c|c|}
\hline Données sociodémographiques & Nombre & Pourcentage (\%) \\
\hline Age & 3 & 8,82 \\
\hline$\leq 30$ & 6 & 17,64 \\
\hline $31-45$ & 19 & 55,88 \\
\hline $46-60$ & 4 & 11,76 \\
\hline $61-75$ & 2 & 5,88 \\
\hline$\geq 76$ & & 29,41 \\
\hline Niveau d'étude & 10 & 23,52 \\
\hline Non scolarisé & 8 & 44,11 \\
\hline Primaire & 15 & 2,94 \\
\hline Secondaire & 1 & \\
\hline Universitaire & & 17,64 \\
\hline Situation sociale & 6 & 20,58 \\
\hline Fonctionnaire & 7 & 23,52 \\
\hline Ménagère & 8 & 8,82 \\
\hline Guérisseur & 3 & 14,7 \\
\hline Retraité & 5 & 11,7 \\
\hline Cultivateur & 4 & 2,94 \\
\hline Prestataire de service & 1 & \\
\hline Revendeuse & & \\
\hline
\end{tabular}


Kpabi et al., J. Appl. Biosci. 2020 Etude ethnobotanique des plantes à activités antiparasitaires utilisées en médecine traditionnelle dans la préfecture de Doufelgou au nord du Togo.

\begin{tabular}{|l|c|c|}
\hline Origine du savoir & & \\
\hline Héritage familial & 18 & 51,61 \\
\hline Expérience Personnelle & 11 & 32,26 \\
\hline Héritage familial + Expérience Personnelle & 5 & 16,13 \\
\hline Raison de la phytothérapie & 18 & 52,94 \\
\hline Coutume & 12 & 35,29 \\
\hline Faible coût & 2 & 5.88 \\
\hline Efficacité & 2 & 5.88 \\
\hline Complémentaire & & \\
\hline Ethnie & 16 & 47,06 \\
\hline Nawd'ba & 17 & 50,00 \\
\hline Lamba & 1 & 2,94 \\
\hline Kotokoli & & \\
\hline
\end{tabular}

Diversité des plantes collectées: A l'issue de cette enquête, 50 espèces appartenant à 28 familles et 46 genres ont été recensées (Tableau 3). Les familles les plus représentées étaient celles des Fabaceae avec 6 espèces suivie des Asteraceae et des Rutaceae avec 4 espèces chacune (Figure 2).

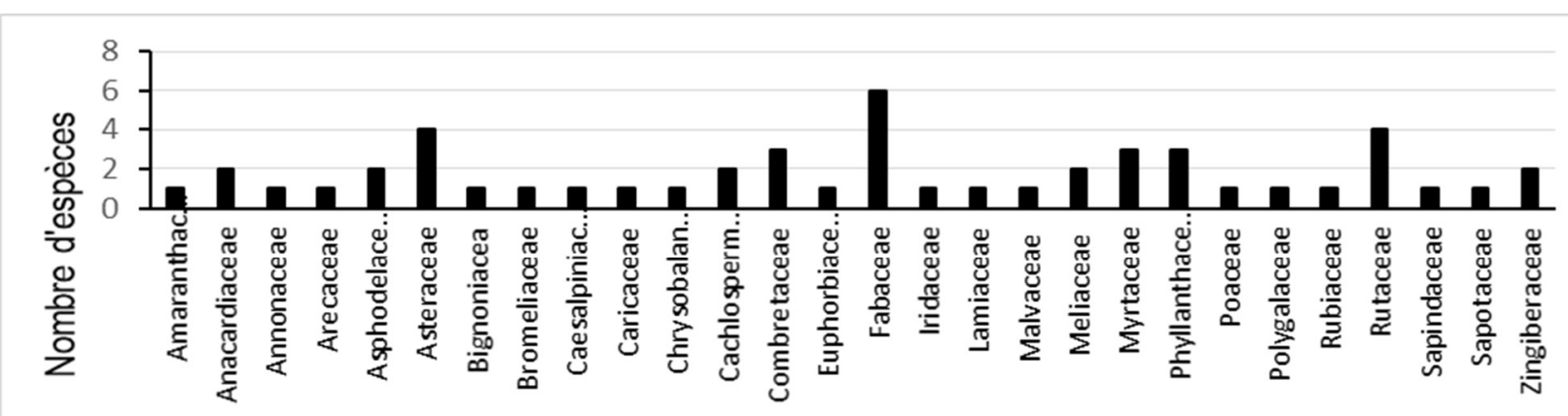

Familles

Figure 2 : Liste des familles des plantes recensées.

Parmi ces plantes, 29 espèces ont été mentionnées pour leur utilisation contre le paludisme. Les plantes les plus citées pour leur utilisation contre le paludisme étaient : Newbouldia laevis P. Beau (26,47\%), Aloe buettneri A. Berger (17,64\%), Carica papaya L. $(14,7)$ et Sarcocephalus latifolius (Sm.) E. A. Bruce $(11,76 \%)$. Les enquêtés ont mentionné 10 plantes dans le traitement des vers intestinaux. Euphorbia hirta L. (38,23\%), Cassia sieberiana DC. $(32,35 \%)$ et Carica papaya L. $(26,47 \%)$ étaient les plus citées pour leur utilisation contre les nématodes. Pour le traitement de la dysenterie amibienne, 25 plantes ont été citées parmi lesquelles nous avons Flueggea virosa (Roxb. Ex Will) Voigt $(26,47 \%)$, Khaya senegalensis (Desr.) A. Jus $(14,70 \%)$, Citrus limon (L.) Burn.f. $(17,7 \%)$ et Phyllanthus amarus Schumach. \& Thon (8.88\%).

Facteur de consensus d'utilisation: Le facteur de consensus d'utilisation des plantes dans le traitement des maladies parasitaires étudiées est présenté au tableau 2. Parmi ces maladies, les informateurs avaient un plus grand consensus pour le traitement de vers intestinaux $(F C=0,83)$ suivi par le paludisme $(F C=0,63)$ et enfin l'amibiase $(F C=0,56)$.

Tableau 2 : Facteur de consensus d'utilisation des informateurs pour les maladies traitées

\begin{tabular}{|l|c|c|c|}
\hline Maladies & Nombre d'utilisation & Nombre total d'espèces citées & FC $^{*}$ \\
\hline Paludisme & 77 & 29 & 0,63 \\
\hline Vers & 56 & 10 & 0,83 \\
\hline Amibiase & 56 & 26 & 0,56 \\
\hline
\end{tabular}

FC : facteur de consensus 
Parties de plantes utilisées, mode de préparation et voies d'administration des recettes: Les personnes enquêtées utilisent différentes parties des plantes pour la préparation des remèdes. Les feuilles $(22,91 \%)$ et les écorces de tiges $(21,35 \%)$ étaient les parties les plus utilisées (Figure 3). Les autres parties utilisées sont : la plante entière $(13,02 \%)$, racines $(11,97 \%)$, écorces de racines $(7,81 \%)$, graines $(7,81 \%)$, bulbes $(6,25 \%)$, jus $(3,64 \%)$, fruit $(3,12 \%)$ et la fleur $(2,08 \%)$. Ces parties de plantes (Figure 4) sont préparées principalement sous forme de décocté $(47,91 \%)$ et de macération $(40,62$ $\%)$ puis administrées essentiellement par voie orale $(84,81 \%)$ (Figure 5).

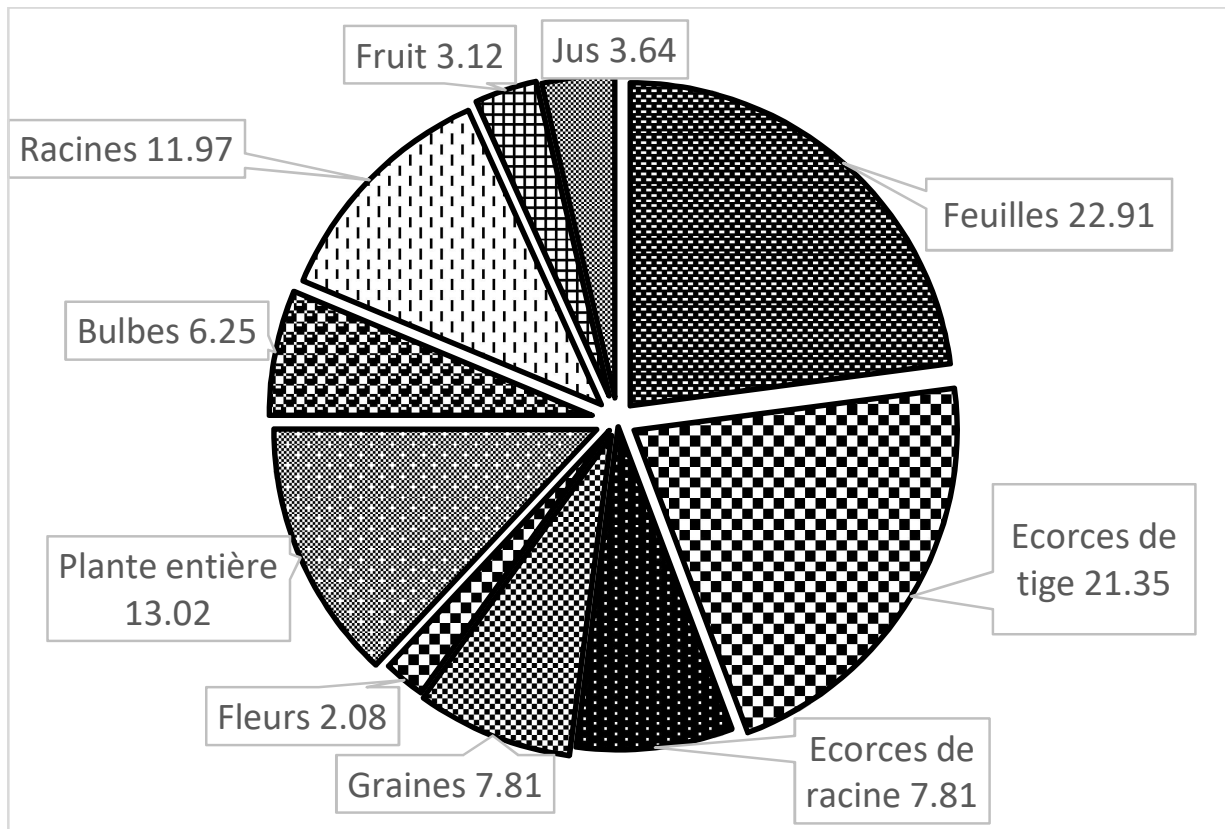

Figure 3 : Les parties de plantes utilisées 


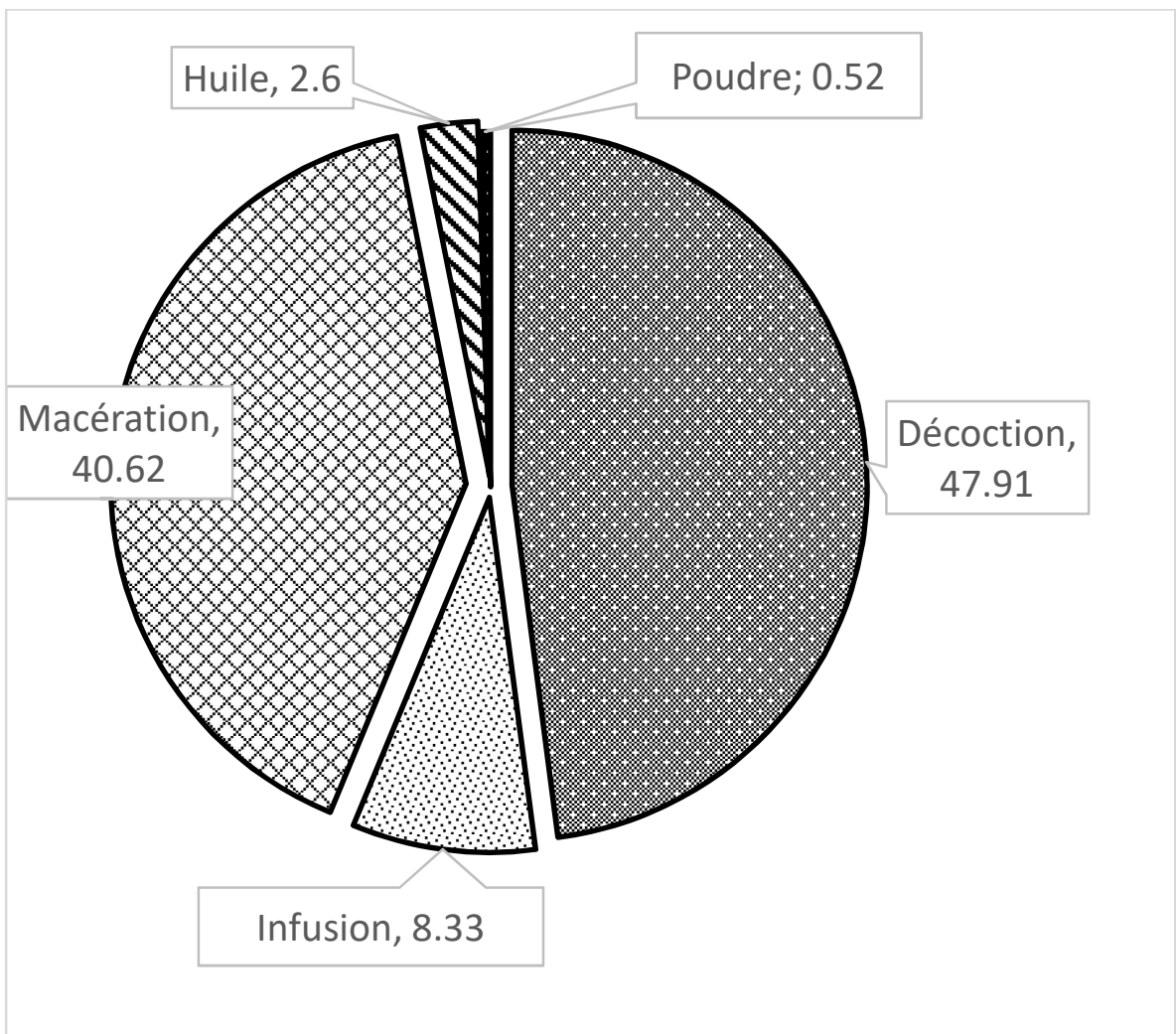

Figure 4 : Mode d'obtention de la drogue

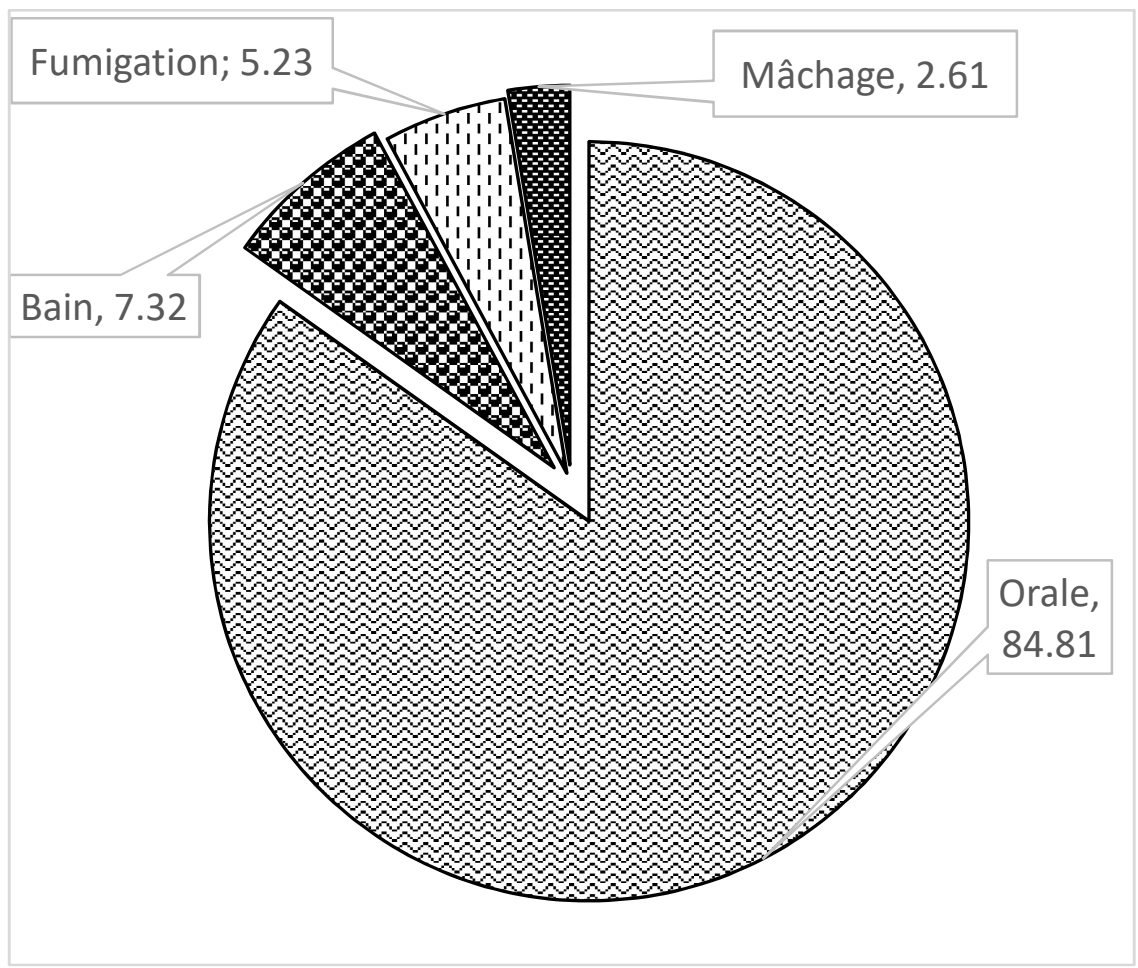

Figure 5 : Voies d'administration des drogues 


\section{DISCUSSION}

La présente enquête documente les plantes médicinales traditionnelles couramment utilisées pour le traitement des maladies parasitaires dans la préfecture de Doufelgou au Togo. La première observation de cette étude est que la majorité des enquêtés étaient des hommes. Ce profil est typique de la plupart des enquêtes réalisées chez les tradithérapeutes. Ceci indique que la pratique de la médecine traditionnelle est beaucoup plus réservée aux hommes. La majorité des personnes enquêtées avait plus de 46 ans. En effet, les personnes âgées sont censées fournir des informations plus fiables, du fait qu'elles détiennent une bonne partie du savoir ancestral qui se transmet oralement (Lakouéténé et al., 2009; Gnagne et al., 2017). Cette connaissance des plantes pourrait être perdue si la nouvelle génération ne prend pas la relève. II est donc important d'enregistrer les médicaments traditionnels et de les valider afin de donner plus de crédit à ces connaissances (Danton et al., 2019). Concernant l'origine du savoir, 52,94 \% des personnes enquêtées ont été initiées au sein de la famille. Sema et al., (2018) ont remarqué après leur enquête réalisée dans la préfecture de Doufelgou que 83 $\%$ des personnes enquêtées avaient hérité cette pratique de leurs ascendants. La transmission de connaissances se fait soit en lignée matrimoniale ou patrimoniale sans distinction de sexe ni d'âge. La médecine traditionnelle est utilisée depuis des générations et est transmise d'une génération à l'autre. Le fait de se soigner avec les plantes est devenu culturel à tel point que les indigènes préfèrent se soigner avec des plantes plutôt que d'aller à l'hôpital. De plus, les populations vivant dans les zones rurales des pays en développement tel que le Togo ont un accès difficile à la médecine moderne. De ce fait, ils se soignent par les plantes (Karou et al., 2011; Kpodar et al., 2015; Adebayo and Amoo, 2019). Concernant la diversité des espèces de plantes collectées, les familles les plus représentées étaient celle des Fabaceae suivie des Asteraceae, Combretaceae, Myrtaceae et Rutaceae. Sema et al., (2018) après une enquête chez des familles spécialisées dans le traitement de diverses pathologies dans la même préfecture ont trouvé que les espèces appartenant à la famille des Fabaceae étaient les plus utilisées ensuite viennent les Euphorbiaceae, les Lamiaceae et les Rutaceae. Les plantes les plus citées pour leur utilisation contre le paludisme étaient : Newbouldia laevis, Aloe buettneri, Carica papaya, Mangifera indica et Sarcocephalus latifolius dont les fréquences de citation sont respectivement : $26,47 \%$; $17,64 \% ; 14,7 \% ; 14,7 \%$ et $11,76 \%$. Les familles les plus représentées dans le traitement du paludisme étaient les Fabaceae (4 espèces), Rutaceae et Combretaceae contenant 3 espèces chacune. Une enquête réalisée dans la région Maritime au Togo a démontré que les Rubiaceae et Rutaceae sont les familles contenant le plus d'espèces utilisées contre le paludisme. Les Rubiaceae sont souvent citées en pharmacologie car elles regorgent des espèces telles que Sarcocephalus latifolius et Morinda lucida Benth. qui contiennent des métabolites secondaires (terpénoides et alcaloïdes indoliques) exerçant des activités antiplasmodiales (Koudouvo et al., 2011). Des tests in vitro réalisés avec les extraits de feuilles de Newbouldia laevis ont démontré une inhibition de Plasmodium falciparum de façon dose dépendante avec une $\mathrm{IC}_{50}$ de $12,6 \mu \mathrm{g} / \mathrm{ml}$ (Gbeassor et al., 1990). Un pigment couplé naphtoquinone-anthraquinone nommé newbouldiaquinone $A$, isolé de Newbouldia laevis a montré une chimiosuppression modérée de la croissance de Plasmodium (Eyong et al., 2006). L'activité antiparasitaire de Carica papaya a également été prouvée par des études in vitro (Kovendan et al., 2012). Des études révèlent que Acanthospermum hispidum DC de la famille des Asteraceae contient des lactones sesquiterpéniques responsables de son activité antiplasmodiale (Ganfon et al, 2012). Parmi les plantes recensées, 10 sont mentionnées pour leur utilisation contre les vers intestinaux. Euphorbia hirta $(38,23 \%)$, Cassia sieberiana (32,35 \%) et Carica papaya $(26,47 \%)$ étaient les plus citées. Des études ont démontré l'activité anthelminthique de Euphorbia hirta (Adedapo et al., 2005), de Carica papaya (Satrija et al., 1995) et de Cassia sieberiana (Diehl et al., 2004 ; Waterman et al., 2010). Cette activité serait due à la présence de polyphénols tels que les tannins (Brunet et al., 2008). Pour le traitement de la dysenterie amibienne, 25 plantes ont été citées parmi lesquels nous avons Flueggea virosa $(26,47 \%)$, Khaya senegalensis $(14,70 \%)$, Citrus limon (17,7\%) et Phyllanthus amarus (8,88 \%). L'huile essentielle de Citrus limon a démontré une inhibition avec pour concentration minimale létale de $1,125 \mu \mathrm{l} / \mathrm{ml}$ après 24 heures sur des flagellés intestinaux (Zenner et al., 2003). Les informateurs avaient un consensus plus élevé pour le traitement des vers intestinaux ( $F C=0,83)$ suivit par le paludisme $(F C=0,63)$ et l'amibiase $(F C=0,56)$. Une valeur élevée (proche de 1) indique qu'un nombre réduit d'espèces bien connues est utilisé par une grande partie des guérisseurs pour soigner une maladie en particulier. Par contre, une valeur faible indique que les informateurs ne sont pas 
d'accord avec l'utilisation de ces espèces. D'après la littérature, des valeurs de FC comprises entre 0,25 et 0,73 sont significatives (Heinrich et al., 1998 ; Umair et al., 2017). Les valeurs du facteur de consensus obtenues après cette étude prouvent que les enquêtés sont d'accord sur les plantes utilisées dans le traitement des vers intestinaux, du paludisme et de l'amibiase. Cela démontre une maîtrise du traitement de ces 3 pathologies. Ces connaissances endogènes peuvent donc servir de base pour la promotion de la médecine traditionnelle au Togo. Divers organes de ces plantes sont utilisés dans la préparation des remèdes. Les feuilles sont les parties de plantes les plus utilisées. Plusieurs auteurs dans leurs travaux ont trouvé également que les feuilles étaient les parties les plus utilisées (Lakouéténé et al., 2009 ; Hoekou et al., 2016 ; Manzo et al., 2017). En effet, le prélèvement des feuilles ne présente pas de danger pour la plante. D'après

\section{CONCLUSION ET APPLICATION DES RÉSULTATS}

L'enquête effectuée dans la préfecture de Doufelgou a permis de recenser 50 espèces de plantes médicinales utilisées couramment dans le traitement des maladies parasitaires. Ces résultats constituent une base de certains auteurs, le prélèvement de $50 \%$ des feuilles d'une plante n'affecte pas de façon significative la survie de cette dernière contrairement aux écorces et racines (N'Guessan et al., 2009). Les feuilles sont des sources primaires d'organes de photosynthèse. Elles synthétisent des exsudats contenant des métabolites secondaires bioactifs qui protègent la plante contre les agressions extérieures. Ce sont ces métabolites qui ont une valeur médicinale pour l'Homme (Agbodeka et al., 2017). Ces parties de plantes sont préparées principalement sous forme de décocté et de macération. La décoction permet de recueillir le plus de principes actifs et atténue ou annule l'effet toxique de certaines recettes; ce qui explique l'utilisation préférentielle de cette méthode comparée aux autres méthodes d'extraction (Lakouéténé et al., 2009; Gnagne et al., 2017 ; Manzo et al., 2017).

données qui permettra la sélection des plantes pour la recherche de nouvelles molécules à activité anthelminthique.

\section{REMERCIEMENT}

Nous tenons à remercier les tradipraticiens et personnes ressources de la préfecture de Doufelgou pour avoir partagé leurs connaissances traditionnelles.

\section{REFERENCES BIBLIOGRAPHIQUES}

Adebayo S.A, Amoo SO, 2019. South African botanical resources: A gold mine of natural proinflammatory enzyme inhibitors ? South African Journal of Botany 123: 214-227.

Adedapo AA, Shabi, OO, Adedokun OA, 2005. Anthelmintic efficacy of the aqueous crude extract of Euphorbia hirta Linn in Nigerian dogs. Vet Arhiv 75: 39-47.

Agbodeka K, Gbekley HE, Karou SD, Anani K, Simpore J, 2017. Activité antiplasmodiale des plantes médicinales d'Afrique de l'Ouest: Revue de la littérature [Antimalarial activity of medicinal plants from West Africa: A review]. 28: 9.

Brunet S, Jackson F, Hoste H, 2008. Effects of sainfoin (Onobrychis viciifolia) extract and monomers of condensed tannins on the association of abomasal nematode larvae with fundic explants. International Journal for Parasitology 38: 783-790.

Danton O, Somboro A, Fofana B, Diallo D, Sidibé L, Rubat-Coudert C, Marchand F, Eschalier A,
Ducki S, Chalard P, 2019 Ethnopharmacological survey of plants used in the traditional treatment of pain conditions in Mali. Journal of Herbal Medicine: 100271.

Diehl MS, Atindehou KK, Téré H, Betschart B, 2004. Prospect for anthelminthic plants in the Ivory Coast using ethnobotanical criteria. Journal of Ethnopharmacology 95: 277-284.

Eyong KO, Folefoc GN, Kuete V, Beng VP, Krohn K, Hussain $H$, Nkengfack AE, Saeftel M, Sarite SR, Hoerauf A, 2006. Newbouldiaquinone A: A naphthoquinone-anthraquinone ether coupled pigment, as a potential antimicrobial and antimalarial agent from Newbouldia laevis. Phytochemistry 67: 605-609.

Ganfon H, Bero J, Tchinda AT, Gbaguidi F, Gbenou J, Moudachirou M, Frédérich M, Quetin-Leclercq $\mathrm{J}$, 2012. Antiparasitic activities of two sesquiterpenic lactones isolated from Acanthospermum hispidum D.C. Journal of Ethnopharmacology 141: 411-417. 
Gbeassor M, Kedjagni AY, Koumaglo K, de Souza C, Agbo K, Aklikokou K, Amegbo KA, 1990. In vitro antimalarial activity of six medicinal plants. Phytotherapy Research 4: 115-117.

Gnagne AS, Camara D, Fofie, NBY, Bene K, Zirihi GN, 2017. Étude ethnobotanique des plantes médicinales utilisées dans le traitement du diabète dans le Département de Zouénoula (Côte d'Ivoire). Journal of Applied Biosciences 113: 11257.

Heinrich M, Ankli A, Frei B, Weimann C, Sticher O, 1998. Medicinal plants in Mexico: Healers' consensus and cultural importance. Social Science \& Medicine 47:1859-71.

Hoekou Y, Tchacondo T, Karou SD, Koudouvo K, Atakpama W, Pissang P, Gbogbo AK, Woegan AY, Batawila K, Akpagana K, Gbeassor M, 2016. Ethnobotanical study of latex plants in the maritime Region of Togo. Pharmacognosy Research 8: 128.

Idowu OA, Soniran OT, Ajana O, Aworinde DO, 2010. Ethnobotanical survey of antimalarial plants used in Ogun State, Southwest Nigeria. African Journal of Pharmacy and Pharmacology 4: 6 .

Karou SD, Tchacondo T, Djikpo Tchibozo MA, AbdoulRahaman S, Anani K, Koudouvo K, Batawila K, Agbonon A, Simpore J, de Souza C, 2011. Ethnobotanical study of medicinal plants used in the management of diabetes mellitus and hypertension in the Central Region of Togo. Pharmaceutical Biology 49: 1286-1297.

Koudouvo K, Karou DS, Kokou K, Essien K, Aklikokou K, Glitho I.A, Simpore J, Sanogo R, De Souza K, Gbeassor M, 2011. An ethnobotanical study of antimalarial plants in Togo Maritime Region. Journal of Ethnopharmacology 134: 183-190.

Koumantiga D, Wala K, Batawila K, Akpagana K, 2013. Les potentialités écotouristiques dans la préfecture de Doufelgou (Togo, Afrique de l'Ouest). Études caribéennes $23: 1-24$.

Kovendan K, Murugan K, Panneerselvam C, Aarthi N, Kumar PM, Subramaniam J, Amerasan D, Kalimuthu K, Vincent S, 2012. Antimalarial activity of Carica papaya (Family: Caricaceae) leaf extract against Plasmodium falciparum. Asian Pacific Journal of Tropical Disease 2: S306-S311.

Kpodar MS, Lawson-Evi P, Bakoma B, Eklu-Gadegbeku K, Agbonon A, Aklikokou K, Gbeassor M, 2015. Ethnopharmacological survey of plants used in the treatment of diabetes mellitus in south of
Togo (Maritime Region). Journal of Herbal Medicine 5: 147-152.

Lakouéténé DPB, Ndolngar G, Berké B, Moyen J-M, KoshKomba E, Zinga I, Silla S, MillogoRasolodimby J, Vincendeau P, Syssa-Magalé L-L, Nacoulma-Ouedraogo OG, Laganier R, Badoc A, Chèze C, 2009. Enquête ethnobotanique des plantes utilisées dans le traitement du paludisme à Bangui. Bull Soc Pharm Bord 148: 123-38.

Manzo LM, Moussa I, Ikhiri K, 2017. Les Plantes Médicinales Utilisées Dans Le Traitement Des Diarrhées Au Niger. Etude Ethnobotanique. Algerian Journal of Natural Products 5:2 475482

Menan ElH, Rouamba E, Ouhon J, Nebavi NGF, Adjetey TAK, 1997. Helminthiases intestinales: résultats de cinq années de coprologie parasitaire. Médecine d'Afrique Noire : 5.

N'Guessan K, Tra Bi FH, Koné MW, 2009. Étude ethnopharmacologique des plantes antipaludiques utilisées en médecine traditionnelle chez les Abbey et Krobou d'Agboville (Côte-d'Ivoire). Ethnopharmacologia 44: 42-50.

Niaré DS, Aissata O, Didier D, Tran TM, Abdrahamane $\mathrm{T}$, Jules S, Kassoum K, Compton P, Boubacar T, Doumbo OK, 2018. Prévalences, du paludisme, des parasitoses intestinales et urinaires à Kalifabougou, Mali. Mali Médical 33.

Ouachinou JM-AS, Dassou GH, Idohou R, Adomou AC, Yédomonhan $\mathrm{H}, 2019$. National inventory and usage of plant-based medicine to treat gastrointestinal disorders with cattle in Benin (West Africa). South African Journal of Botany 122: 432-446.

Satrija F, Nansen P, Murtini S, He S, 1995. Anthelmintic activity of papaya latex against patent Heligmosomoides polygyrus infections in mice. Journal of ethnopharmacology 48: 161-164.

Sema M, Atakpama W, Kanda M, Koumantiga D, Batawila K, Akpagana K, 2018. Une forme de spécialisation de la médecine traditionnelle au Togo: Cas de la préfecture de Doufelgou. Journal de la Recherche Scientifique de I'Université de Lomé 20: 29-43-43.

Umair M, Altaf M, Abbasi AM, 2017. An ethnobotanical survey of indigenous medicinal plants in Hafizabad district, Punjab-Pakistan. PLOS ONE 12-:6 e0177912. 
Kpabi et al., J. Appl. Biosci. 2020 Etude ethnobotanique des plantes à activités antiparasitaires utilisées en médecine traditionnelle dans la préfecture de Doufelgou au nord du Togo.

Waterman C, Smith RA, Pontiggia L, DerMarderosian A, 2010. Anthelmintic screening of Sub-Saharan African plants used in traditional medicine. Journal of ethnopharmacology 127: 755-759.

Zenner L, Callait MP, Granier C, Chauve C, 2003. In vitro effect of essential oils from Cinnamomum aromaticum, Citrus limon and Allium sativum on two intestinal flagellates of poultry, Tetratrichomonas gallinarum and Histomonas meleagridis. Parasite 10: 153-157. 
Tableau 3 : Liste de plantes recensées

\begin{tabular}{|c|c|c|c|c|c|c|c|c|}
\hline \multirow[t]{2}{*}{ Famille } & \multirow[t]{2}{*}{ Nom scientifique } & \multirow[t]{2}{*}{ Nom local } & \multirow{2}{*}{$\begin{array}{l}\text { Partie } \\
\text { utilisée }\end{array}$} & \multirow{2}{*}{$\begin{array}{l}\text { Mode de } \\
\text { préparation }\end{array}$} & \multirow[t]{2}{*}{ Administration } & \multicolumn{3}{|l|}{ FRC } \\
\hline & & & & & & Paludisme & Vers & Amibiase \\
\hline Amaranthaceae & Chenopodium ambrosioides $\mathrm{L}$. & Moyeye bgna & $\mathrm{F}$ & Macération & VO & - & 2,94 & - \\
\hline \multirow[t]{2}{*}{ Anacardiaceae } & Anacardium occidentale L. & $\begin{array}{l}\text { Anansara- } \\
\text { kpagna }\end{array}$ & ET & Décoction & VO & 5,88 & 2,94 & - \\
\hline & Mangifera indica L. & Moungou & ET & Décoction & VO & 14,7 & - & - \\
\hline Annonaceae & Annona senegalensis Pers. & Amoumode & $\mathrm{F}$ & Décoction & VO & 2,94 & - & - \\
\hline Arecaceae & Cocos nucifera L. & $\begin{array}{l}\text { Anansara- } \\
\text { kpakpar }\end{array}$ & Noix & Infusion & VO & 2,94 & - & - \\
\hline \multirow[t]{2}{*}{ Asphodelaceae } & Aloe buettneri A. Berger & $\begin{array}{l}\text { Naa } \\
\text { Nansoulmre }\end{array}$ & $\mathrm{R}$ & Décoction & VO & 17,64 & - & - \\
\hline & Aloe vera (L) Burm.f. & Aloes & $\mathrm{F}$ & Macération & VO & - & & 2,94 \\
\hline \multirow[t]{4}{*}{ Asteraceae } & Acanthospermum hispidum DC, & $\begin{array}{l}\text { Kpan'na } \\
\text { Gnirikou }\end{array}$ & $\mathrm{F}$ & Décoction & VO & 5,88 & - & 2,94 \\
\hline & Artemisia absinthium L & - & $\mathrm{F}$ & Décoction & VO & - & - & 2,94 \\
\hline & Tridax procumbens $\mathrm{L}$. & Sakrpia & PE & Décoction & VO & - & - & 2,94 \\
\hline & Vernonia amygdalina Delile & Souwaka & $\mathrm{F}$ & Macération & VO & - & 2,94 & 2,94 \\
\hline Bignoniaceae & Newbouldia laevis P. Beauv. & Kpatimé & $\mathrm{F}$ & Décoction & VO & 26,47 & - & - \\
\hline Bromeliaceae & Ananas comosus (L.) Merr. & Atoto & $\mathrm{Fr}$ & Décoction & VO & 2,94 & - & 2,94 \\
\hline Caesalpiniaceae & Daniellia oliveri (Rolfe) Hutch. \& Dalziel & Tchade & $\mathrm{F}$ & Décoction & Bain & 2,94 & - & - \\
\hline Caricaceae & Carica papaya $\mathrm{L}$ & Adiba, Adibgou & $\mathrm{Fr}$ & Macération & VO & 14,7 & 26,47 & - \\
\hline Chrysobalanaceae & Parinari curatellifolia Planch. ex Benth. & $\begin{array}{l}\text { Fekourou } \\
\text { Fekourkou }\end{array}$ & ET & Macération & VO & - & - & 2,94 \\
\hline \multirow[t]{2}{*}{ Cachlospermaceae } & Cochlospermum planchonii Hook. F. & Kalaahoka & $\mathrm{R}$ & Décoction & VO & - & - & 2,94 \\
\hline & $\begin{array}{l}\text { Cochlospermum tinctarium Perr. ex } \\
\text { A.Rich. }\end{array}$ & Kalaahoka & $\mathrm{R}$ & Décoction & VO & - & - & 5,88 \\
\hline \multirow[t]{3}{*}{ Combretaceae } & Combretum micranthum G. Don & Wonwon & $\mathrm{F}, \mathrm{R}$ & Décoction & VO & 5,88 & - & 5,88 \\
\hline & Pteleopsis suberosa Engl. \& Diels & Kassissigna & ET & Décoction & VO & 5,88 & - & - \\
\hline & Terminalia laxiflora Engl. \& Diels & Atcharkpantand & $\mathrm{ET}, \mathrm{F}$ & Décoction & VO, Bain & 2,94 & - & - \\
\hline Euphorbiaceae & Euphorbia hirta L & Tchirm-tchirm, & $\mathrm{PE}$ & Macération & VO & - & 38,23 & - \\
\hline \multirow[t]{3}{*}{ Fabaceae } & Cassia siamea Lam. & Acassia & $\mathrm{Fl}$ & Décoction & $\mathrm{VO}, \mathrm{Fu}$ & 8,82 & - & - \\
\hline & Cassia sieberiana DC. & Gnéntou & ET & Macération & VO & - & 32,35 & - \\
\hline & Parkia biglobosa (Jacq.) R.Br. ex G.Don & Soumpire & ET & Décoction & VO & - & & 5,88 \\
\hline
\end{tabular}

Parties utilisées : $F$ : Feuilles, ET : Ecorces de tiges, ER : Ecorces de racines, R : Racine, Fr : Fruits, PE : Plante entière, B : Bulbes, Gr : Graines Voies d'administration : VO : Voie orale, Fu : Fumigation ; FRC : Fréquence relative de citation ; - Non appliqué. 
Kpabi et al., J. Appl. Biosci. 2020 Etude ethnobotanique des plantes à activités antiparasitaires utilisées en médecine traditionnelle dans la préfecture de Doufelgou au nord du Togo.

Tableau 3 : Liste de plantes recensées (suite)

\begin{tabular}{|c|c|c|c|c|c|c|c|c|}
\hline \multirow[t]{2}{*}{ Famille } & \multirow[t]{2}{*}{ Nom scientifique } & \multirow[t]{2}{*}{ Nom local } & \multirow{2}{*}{$\begin{array}{l}\text { Partie } \\
\text { utilisée }\end{array}$} & \multirow{2}{*}{$\begin{array}{l}\text { Mode de } \\
\text { préparation }\end{array}$} & \multirow[t]{2}{*}{ Administration } & \multicolumn{3}{|c|}{ FRC } \\
\hline & & & & & & Paludisme & Vers & Amibiase \\
\hline & $\begin{array}{l}\text { Pericopsis laxiflora (Benth.) } \\
\text { Meeuwen }\end{array}$ & Kedere & ET & Macération & VO & 5,88 & - & 5,88 \\
\hline & $\begin{array}{l}\text { Piliostigma thonningii } \\
\text { (Schumach.) Milne-Redh }\end{array}$ & Pokou & $\mathrm{F}$ & Décoction & Bain & 2,94 & - & - \\
\hline & Tamarindus indica L. & - & $\mathrm{F}$ & Décoction & VO & 2,94 & - & - \\
\hline Iridaceae & $\begin{array}{l}\text { Gladiolus gregarius Welw. ex } \\
\text { Baker }\end{array}$ & Fisoucore & B & Décoction & VO & - & 23,52 & 5,88 \\
\hline Lamiaceae & Ocimum gratissimum $\mathrm{L}$. & Hasou & $\mathrm{F}$ & Macération & VO & - & - & 5,88 \\
\hline Malvaceae & Adansonia diaitata! & 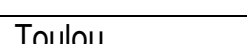 & $\mathrm{Fr}$ & Róroction & VO & - & 2 & 588 \\
\hline \multirow[t]{2}{*}{ Meliaceae } & Azadirachta indica A.Juss. & Niim & $\mathrm{F}$ & Décoction & $\mathrm{Fu}$ & 5,88 & - & \\
\hline & $\begin{array}{l}\text { Kahya senegalensis (Desr.) A. } \\
\text { Juss. }\end{array}$ & Hermou & $\mathrm{ET}, \mathrm{F}$ & $\begin{array}{l}\text { Décoction, } \\
\text { Macération, }\end{array}$ & VO & 8,82 & 8,82 & 14,64 \\
\hline \multirow[t]{3}{*}{ Myrtaceae } & Eucalyptus globulus Labill. & Anansara-tii & $\mathrm{F}$ & Décoction & $\mathrm{VO}, \mathrm{Fu}$ & 8,82 & - & - \\
\hline & Psidium guajava L. & Goyava & $\mathrm{ET}, \mathrm{F}$ & Macération & VO & 5,88 & - & - \\
\hline & $\begin{array}{l}\text { Syzygium aromaticum (L.) Merr. } \\
\text { \& L.M.Perry }\end{array}$ & Atingali & $\mathrm{Fr}$ & Décoction & VO & - & - & 2,94 \\
\hline \multirow[t]{3}{*}{ Phyllanthaceae } & $\begin{array}{l}\text { Flueggea virosa (Roxb. ex Willd.) } \\
\text { Voigt }\end{array}$ & tchaka-tchaka & $\mathrm{R}$ & Décoction & VO, Bain & 2,94 & - & 26,47 \\
\hline & Hymenocardia acida Tul & Kantchila & $\mathrm{F}$ & Décoction & Bain & 2,94 & - & - \\
\hline & $\begin{array}{l}\text { Phyllanthus amarus Schumach. \& } \\
\text { Thonn }\end{array}$ & Gniria & $\mathrm{PE}$ & Décoction & VO & - & 8,82 & 8,82 \\
\hline Poaceae & Zea mays L. & Wamed & $\mathrm{Fr}$ & Poudre & VO & - & - & 2,94 \\
\hline Polygalaceae & $\begin{array}{l}\text { Securidaca } \\
\text { longipedunculata Fresen }\end{array}$ & Poupio & $\mathrm{R}$ & Décoction & VO & - & - & 2,94 \\
\hline Rubiaceae & $\begin{array}{l}\text { Sarcocephalus latifolius (Sm.) E. } \\
\text { A. Bruce }\end{array}$ & Koutoutchalgnisr & $R, F$ & $\begin{array}{l}\text { Macération, } \\
\text { Décoction }\end{array}$ & VO, Bain & 11,76 & - & - \\
\hline
\end{tabular}

Parties utilisées : F : Feuilles, ET : Ecorces de tiges, ER : Ecorces de racines, R : Racine, Fr : Fruits, PE : Plante entière, B : Bulbes, Gr : Graines

Voies d'administration : VO : Voie orale, Fu : Fumigation ; FRC : Fréquence relative de citation ; - Non appliqué. 
Kpabi et al., J. Appl. Biosci. 2020 Etude ethnobotanique des plantes à activités antiparasitaires utilisées en médecine traditionnelle dans la préfecture de Doufelgou au nord du Togo.

Tableau 3 : Liste de plantes recensées (suite et fin)

\begin{tabular}{|c|c|c|c|c|c|c|c|c|}
\hline \multirow[t]{2}{*}{ Famille } & \multirow[t]{2}{*}{ Nom scientifique } & \multirow[t]{2}{*}{ Nom local } & \multirow{2}{*}{$\begin{array}{l}\text { Partie } \\
\text { utilisée }\end{array}$} & \multirow{2}{*}{$\begin{array}{l}\text { Mode de } \\
\text { préparation }\end{array}$} & \multirow[t]{2}{*}{ Administration } & \multicolumn{3}{|c|}{ FRC } \\
\hline & & & & & & Paludisme & Vers & Amibiase \\
\hline \multirow[t]{4}{*}{ Rutaceae } & $\begin{array}{l}\text { Afraegle paniculata (Schumach. } \\
\text { \& Thonn.) Engl. }\end{array}$ & Akoundou & $\mathrm{Gr}$ & Huile & VO & - & 14,7 & - \\
\hline & Citrus limon (L.) Burm.f. & Gnigna-lemou & $\mathrm{R}$, jus & $\begin{array}{l}\text { Infusion, } \\
\text { décoction }\end{array}$ & VO & 11,76 & - & 14,7 \\
\hline & Citrus sinensis (L.) Osbeck & Lemou & $\mathrm{F}$ & Macération & VO & 8,82 & - & - \\
\hline & $\begin{array}{l}\text { Zanthoxylum zanthoxyloides } \\
\text { (Lam.) Zepern. \& Timler }\end{array}$ & Kalwouo & ER & Macération & VO & 5,88 & - & 5,88 \\
\hline Sapindaceae & Blighia sapida K. D. Koenig & Kpissia & ET & Décoction & Bain & 5,88 & - & - \\
\hline Sapotaceae & Vitellaria paradoxa C.F.Gaertn. & Tchoumde & ET & Décoction & VO & - & - & 5,884 \\
\hline \multirow[t]{2}{*}{ Zingiberaceae } & $\begin{array}{l}\text { Aframomum cereum (Hook. f.) K. } \\
\text { Schum. }\end{array}$ & Kperkperou & $B$ & Décoction & VO & 5,88 & - & - \\
\hline & Zingiber officinale Roscoe & Misscore & $\mathrm{R}$ & Décoction & $\mathrm{VO}$ & - & - & 2,94 \\
\hline
\end{tabular}

Parties utilisées : $F$ : Feuilles, ET : Ecorces de tiges, ER : Ecorces de racines, R : Racine, Fr : Fruits, PE : Plante entière, B : Bulbes, Gr : Graines

Voies d'administration : VO : Voie orale, Fu : Fumigation ; FRC : Fréquence relative de citation ; - Non applicable 